\title{
Review
}

\section{Social Determinants of Health and Epigenetics: A New Tool for Health Policy}

\author{
Hitakshi Sehgal and William A. Toscano * \\ Division of Environmental Health Sciences, School of Public Health Sciences, University of Minnesota, \\ Minneapolis, MN 55455, USA; sehg0006@umn.edu \\ * Correspondence: tosca001@umn.edu; Tel.: +1 612-859-2120
}

\begin{abstract}
Globally, humans are struggling with the double threat of communicable and noncommunicable diseases, which are presenting new challenges to public health. Public health problems are generally studied and addressed at primary, secondary and tertiary levels. Most effective results are seen with primary interventions. Public health is becoming more aware of the importance of environment-gene interactions in the ontology of health and disease using epigenetics. Epigenetics is the study of altered gene expression without change in base pairs. Be it physical, social, behavioral, or economic factors; they all influence quality of life and health of individuals and populations. That environments are changing the human health phenotype and these changed phenotypes are heritable is of concern for the future of the human race. Knowing the causes of non-communicable human diseases using epigenetics will contribute to the development of new policies to encourage prevention using primary public health initiatives. Research and application of epigenetics shows great promise for improving population health. Continued advances in epigenetics will enhance how we understand and address the way environments are affecting the human health phenotype. For some time, health systems have been and continue to be tertiary in nature. Epigenetic changes can provide information necessary to better understand how social determinants of health can be used to build societies focused on equitable health for all people, rather than continuing to focus on treatment of diseases in the tertiary phase that leads to health disparities. Understanding mechanisms of social determinants of disease, will allow society to evolve in a health-oriented rather than a disease-oriented world. For this reason, we must enhance and apply epigenetics (physical, social, behavioral, economic) research to policy development. We will discuss how bringing social determinants together with biology can be used as new tools for public health policy.
\end{abstract}

Keywords: social determinants; epigenetics; exposome; public health policy; resilience; allostatic load; adaptation

\section{Introduction}

When the leading risk factors for morbidity and mortality are tabulated, tobacco use, diet and undernutrition, alcohol use, and improper sanitation and clean water are foremost on the list [1]. However, when causes of mortality and morbidity are investigated more deeply, the underlying causes of disease include, poverty, lack of educational opportunities, improper housing, and environments that do not promote health, which include lack of social capital, emerge as the real causes of morbidity and mortality. That the social environment has an impact on the health of populations, has been largely ignored because in practice, most public health efforts are focused on mitigation of specific diseases by medical intervention, and a much smaller effort is focused on identifying causes and prevention of diseases.

Social sciences and biomedical sciences have studied public health in distinct ways, with little communication or collaboration between the two approaches [2]. As the focus of public health shifts toward understanding causes and developing prevention strategies of diseases, there is consensus that integration of diverse approaches is needed to achieve these goals [3]. 
After the sequencing of the human genome in 2000, there was great hope that biological science could find a gene responsible for predicting health and disease outcomes [4]. However, it was soon discovered that even simple human phenotypes such as height of an individual had no specific gene associated with that trait [5].

Epigenetics is the study of heritable changes in gene-expression that occur without changes in DNA sequence [6]. Epigenesis is the biochemical process underlying changes in gene-expression that occur without a change in DNA sequence by activating or silencing specific genes in response to an environmental signal [7]. The environment around the genotype is most critical in determining health phenotype. Epigenesis has provided evidence that the genotype cannot predict the exact phenotype but rather phenotype is controlled by the environment in which the genotype is expressed. Thus the environment in which people live can predict whether they express a health or disease phenotype.

The environment around the gene is not easy to define or study. The environment with which genes interact is known as the exposome [8,9]. The exposome includes nutrients, noise, occupation, psychosocial environment, social economic status and education as well as pollutant chemicals, microorganisms, drugs, food, and additives [9]. The exposome includes social environment as one aspect of the pathway to disease. Wild divided the exposome into three parts, i) general external environment, ii) internal environment, and iii) the specific external environment [9]. The general external environment includes social capital, education, social economic status, and where you live. The internal environment is comprised of metabolism, endogenous hormones, microbial flora, physical activity, and oxidative stress. The specific external environment includes chemical pollutants; infectious agents and life style exposures, such as alcohol and tobacco use [9]. Therefore, the paradigm of exposure $\rightarrow$ physiological change $\rightarrow$ disease, while still true is more complex than previously thought.

Most studies of the exposome and epigenetic expression focus on chemical exposures [10]. However, the social environment is also critical and influences biological processes. In this paper, we will review how the social environment and gene expression are linked, bringing together social and biological sciences as important tools in promoting public health policy.

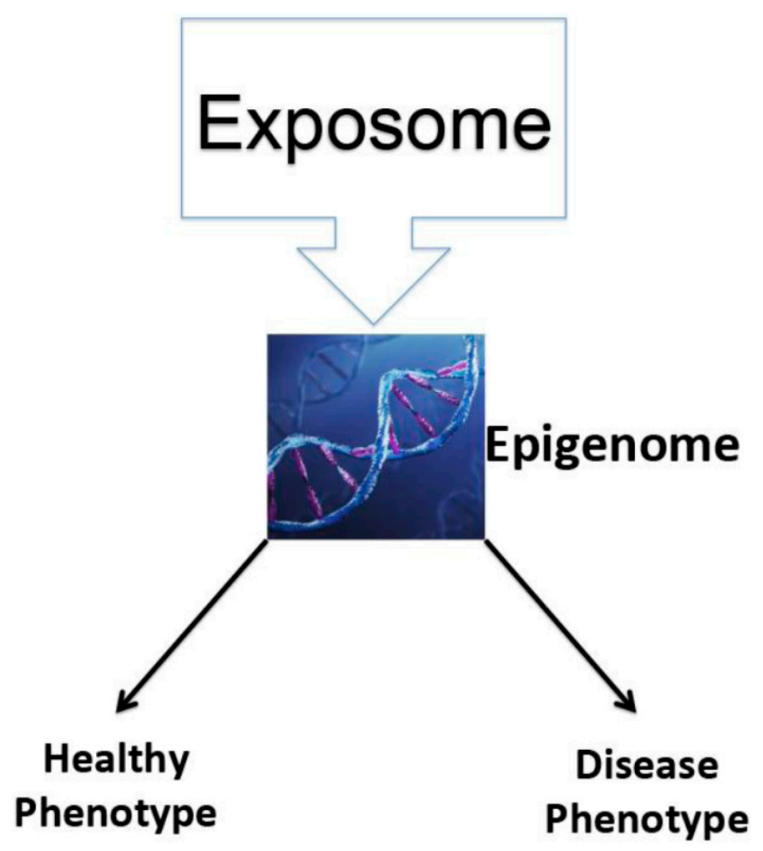

Figure 1. The Exposome and health Outcome. The exposome, comprised of chemical, physical and social environments, including social economic status, social capital, societal inclusion, stress and lifestyles, and living and working conditions interact with the human exposome to alter gene expression. A positive exposome will lead to a healthy phenotype. A negative exposome leads to a disease phenotype. 


\section{Social Epigenetics}

That social factors are involved in health is not a new concept. It has been challenging to adopt an inclusive approach (social, physical, mental) in addressing health issues across the globe. The health system focuses on proximate causes of morbidity and mortality (diseases), including risk factors, while overlooking underlying causes of poor health. So, while tobacco, alcohol, physical inactivity, unhealthy diet, blood glucose, blood cholesterol, and blood pressure are proven risk factors; they fall short of explaining susceptibility differences in populations; of explaining causality, and providing effective strategies for prevention.

Risk factors are not necessarily causal and could themselves be a result of many factors in the exposome acting epigenetically on the body [11]. Research investigating environmental links to disease has helped to establish evidence of how social factors are affecting health of populations. This is important because not only does a social environment rich in resources, affect access to health care and better quality of life, it also seems to have a biological impact leading to better health. Conversely, a resource deprived social environment impedes not only access to health care and basic necessities of life, but also has a biological impact leading to poor health. This explains why emphasizing tertiary health care has little impact on societal health patterns of individuals, communities, and populations [12,13].

\section{Social Capital}

Neighborhoods (communities) are small social regions where people with shared values and concerns interact daily. A conventional sociological explanation for neighborhoods is that people holding similar values cluster together to minimize social conflict. Usually the center of the neighborhood is an area where people gather together and is a focal point for community activities. Ideally, neighborhoods have mixed uses - residential, retail, education, recreation, civic, banking, food, and cultural activities. Neighborhoods could include a well-connected network of safe streets and transportation choices as well as open green spaces. Neighborhoods that work well have room for all - young, old, families, single people, white and blue-collar workers, natives and newcomers. They also have places to go - shops and services, garages, parks, schools, places of worship, and for family and friends to visit. Ideal neighborhoods are high in social capital. Social capital refers to the institutions, relationships, and norms that shape the quality and quantity of a society's social interactions. Increasing evidence shows that social cohesion is critical for societies to prosper economically and for development to be sustainable. Social capital is not just the sum of the institutions, which underpin a society - it is what binds communities together [14]. Social capital is the expected collective benefit derived from the preferential treatment and cooperation between individuals and groups. There are many components to social capital, including, connections between people, social networks, reciprocity, trust, social connections between family, friends, and neighbors [14]. Social networks have value because they increase connectedness. Social networks include strong ties (family) and weak ties (membership in clubs, unions, neighbors), both of which are important to well working networks. Face-to-face ties are most important in a well-functioning social network. Social networks benefit individuals, for example when neighbors help one another when a person in the household is ill, or otherwise incapacitated. Positive social capital comprises mutual support, cooperation, trust, and institutional effectiveness. Negative social capital, however, leads to sectarianism, corruption, and ethnocentrism, which are characterized by prejudice, racism, and hostile environments $[15,16]$. Social capital and social networks, not external agencies such as NGO's or charities, will empower people to take charge of their own health.

Communities with high social capital tend to have better health. This is sometimes referred to as the Roseto effect [17]. Roseto is a community of Italo-Americans in Eastern Pennsylvania. This community had a low level of deaths by myocardial infarction when compared to local surrounding communities that shared the same water supply, physicians and health care facilities [18], in spite of exposure to today's most common risk factors [17]. The community had a stable structure that emphasized family, a supportive social network, and a high level of ethnic homogeneity. In the late 1960s, this community with high social capital changed. The younger generation started to move 
away from the community, and assumed a more assimilated life style, social capital in the community diminished, accompanied with an increase in deaths from heart disease $[17,18]$. Some investigators sought to determine whether the observed effects were genetically regulated. The data suggest, it was more than just ethnicity that had a protective effect on the health of people from Roseto, because other populations of Italo-Americans living a more assimilated lifestyle had disease profiles similar to the general population [19]. The observed health implications of high or low social capital have an effect on health that cannot be explained by the genome exclusively, thus, it is likely a process that acts above the genome such as epigenetics in modulating these effects [11,20].

\section{Social Environment and Health:}

Interesting data were obtained in a study of cardiovascular diseases (CVD) in British civil servants. In that study, the investigators showed that there were marked differences in health outcomes related to job classification [21]. Employees of higher rank showed better health outcomes than those of lower rank, even though all employees had equal access to health care [22]. In addition, other studies show lifetime health effects resulting from early life exposure to stress [21-24].

This helps us connect social factors to health outcomes and makes us rethink the current perception of health behaviors. Health behaviors and disease outcomes are often weakly correlated but easily generalized. So for example, obesity is often attributed to diet and lack of exercise when the true cause may be an underlying environmental factor. Studies in primates show that sociocultural factors affect health-behavior. Social class, parenting, social integration, social isolation, and social defeat were some factors that were studied and linked to deleterious health effects, weight gain, stereotyped behavior, and anti-social tendencies [23,25]. Thus, weight gain could be an effect of social isolation or another environmental trigger but we generalize it to lack of exercise and diet. Additionally, similar behavior in different communities and groups is ignored and remains unexplained. For example, why does food or exercise not affect everyone in a similar manner? Similarly poor health has also been compartmentalized where it is thought that chronic diseases such as diabetes and obesity are diseases affecting only high social economic status (SES) groups, or infectious diseases such as tuberculosis affect only the poor, until data that suggested otherwise became available [26]. Much of health policy is dictated by such generalizations because of lack of understanding of causality. Health policy has been largely focused on tertiary treatment of disease and development of drugs to mitigate the effects of common diseases. However, very little funding or attention has focused on primary disease prevention strategies. It is time that policy makers take into account advances in epigenetic science to understand underlying causes of diseases so that proper prevention strategies may be undertaken. For example, it is an easy supposition that lack of exercise and bad food choices cause obesity and related diseases, however, it is not that simple. This is also the reason that health policy fails to address primary prevention and relies on access to expensive lifestyles or healthcare. Epigenetics reveals that health behaviors cannot be generalized but the only generalization is environmental.

The world shares the environment. As we focus on populations, populations share common geographical areas and physical environments. Communities share socio-cultural-political environments. Families learn and practice these social- cultural activities [26]. Some of the community norms include how and where people live and what they eat, the respective roles of menwomen, old- young-children and individuals who are at the bottom of the community. The genome at the core of the individual, lies ready to be influenced by the larger environment and will produce the phenotype best suited to that environment. The brain is continuously receiving these environmental influences and signaling the gene to produce a phenotype 'fit to survive' (Figure 1). Among many of its functions, the brain is 'social'[27]. The genome of individuals in a population shares $99.9 \%$ congruence. The other 0.1 percent is thought to determine susceptibility of the genome to environmental insults through single nucleotide polymorphisms (SNPs) [28]. [29]

With globalization, the world shares a lot of socio-political and physical environments. Studies have limited public health professionals to addressing related factors rather than addressing the real 
causes of poor health. Thus, we continue to research within limitations, confounding for social factors and looking for easier solutions rather than finding real solutions [30].

Epigenetics shows us that the human body is continually communicating with the exposome and responding to it. This interaction may or may not be advantageous. Biomedical science has focused on the changes happening within the body. Social science has focused on individuals living as populations, their interactions, differences and similarities. Findings from these dichotomous endeavors have prompted arguments about nature versus nurture as factors determining human health or disease [31], although the border between the two views is becoming less rigid. The body is continuously receiving signals from the exposome and these signals are transmitted to various organs through epigenetic modification. For example, the food we eat contains both nutrients and potential contaminants that are signals to which the body will respond. Social interactions with family, community, and the world are also signals to which the body will respond. Epigenetics can explain the underlying mechanisms of responses in both cases.

Finding causes for common complex diseases is not an easy task. Common diseases have multiple factors involved in the onset and development of the disease. Often environment is an underlying factor that brings about the change in health phenotype. Stress can be both a cause and an outcome of disease. Epigenetic studies have started to shed new light in the development of common diseases, which has forced us to shift our thinking about disease etiology. Several genes had been thought to be associated with antisocial behavior and poor self-control in children when they were reared in poor parenting situations, however the variances were not associated with poor selfcontrol, but how the social environment affects children's behavior. Some individuals are deeply affected whereas others are not [29,32]. Finding the reasons for the differences will involve an epigenetic approach. The skeletal, muscular, nervous, respiratory, cardiovascular, digestive, excretory, endocrine, immune, integumentary, and reproductive systems are all necessary for existence. Acute or chronic insults to these systems, such as war, infections, natural and anthropogenic disasters, severe injuries can threaten survival, but also may be processed by the body and evoke epigenetic responses.

Epigenetic changes are consequences of these inputs from the exposome. They may remain or be reversed via other interactions [33]. For example, environmental signals from living in an unsafe neighborhood are perceived as a threat to survival. The stress resulting from this perceived threat to personal safety evokes a response of fear leading the person to either flee or fight the situation, driven by corticosterones and catecholamines [29]. Ideally, this response subsides after the threat has elapsed (Figure 2). In some people, the inactivation of this response to stress can be slow leading to lingering of stress hormones in the body. Thus, the body is overexposed to stress hormones, leading to a situation called allostatic load [29]. Allostatic load can arise in a variety of situations such as: i.) Slow inactivation and lingering of stress hormones, ii.) Frequent stress, iii.) Lack of adaptation to repeated stressors of the same kind and, iv.) Lack of activation of allostatic systems leading to activation of other inflammatory responses that have deleterious effects on the body. A prolonged allostatic (adaptive) load can bring about physiological conditions leading to chronic illness such as diabetes, obesity, CVD or other chronic conditions.

Many environmental factors could contribute to the allostatic load of individuals. An individual often has little or no control over the factors causing allostatic load. For example, an individual has little or no control over smoking or second-hand tobacco smoke, alcohol consumption, unhealthy food, sedentary lifestyle, polluted air, pesticide-laden foods, and unsafe neighborhoods or work environments. The individual has no control over how the body reacts to the stresses that those exposures generate. 


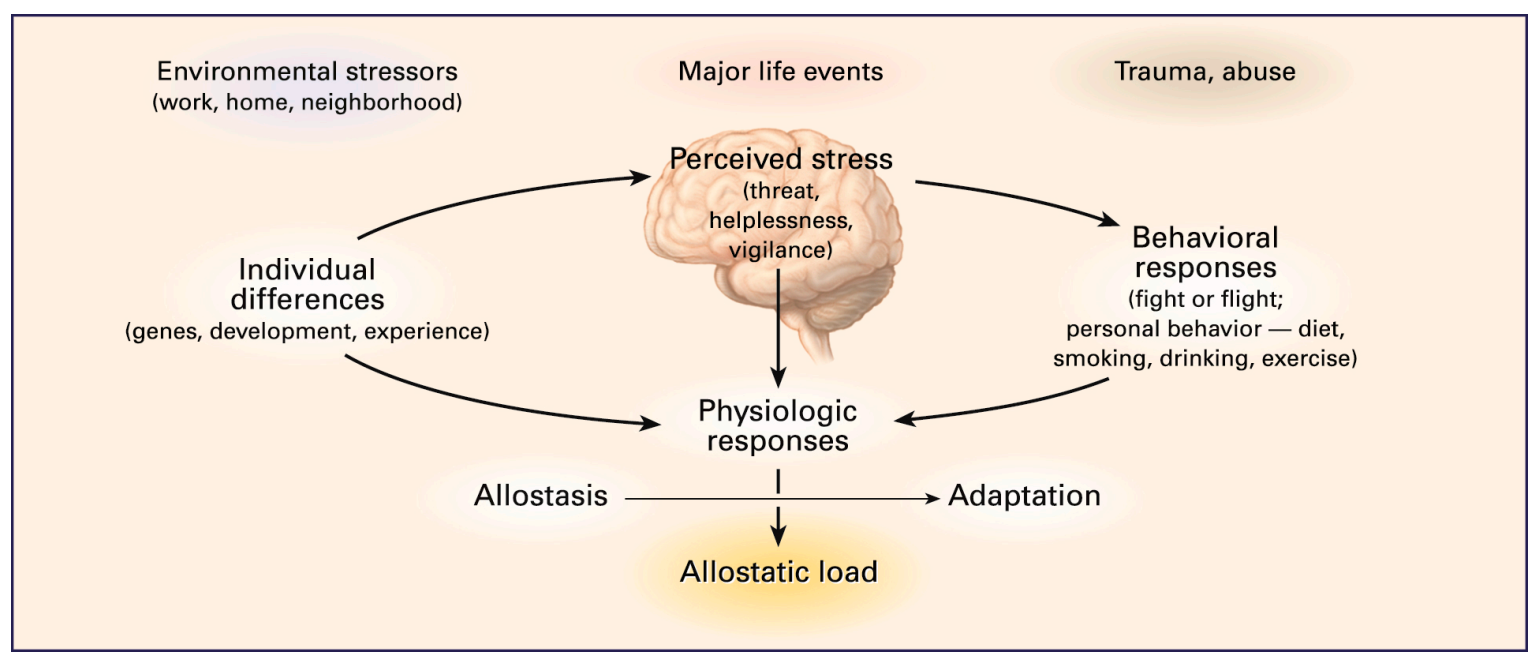

Figure 2. The effect of stress on allostatic load. When individuals or communities are exposed is exposed to repeated or chronic stressors from the environment, and life altering events physiological and behavioral changes occur in the ability to manage those stresses. Those with high allostatic load demonstrate slow resilience to the stressors, which may modify the exposome to lead to shorter lifespans or onset of chronic diseases in the population. Reprinted with permission from [29], copyright Massachusetts Medical Society.

The exposure to risk factors and access to a healthy exposome is controlled largely by public health policy. Populations are exposed to common stressors because of a lack of policies that address a healthy exposome. This leads to many people developing an allostatic load from common stressors with the population developing a disease pattern. If the population comes together (social capital) to address these stressors and bring about positive changes through policy, then common stressors would be minimized, which would promote a healthy exposome. Thus the mitigation of allostatic load in a population is directly correlated with positive social capital to promotes health for all. The lower the number of stressors that a population has, the better the health. Because the global focus of health has been on tertiary care we are not alleviating allostatic loads in populations. Only preventive care will accomplish this. Cumulative allostatic loads, which are higher in underserved communities, lead to premature illness and death. If this is not addressed by policy, then the cumulative allostatic loads results in a high population stress load, as we see in low and middleincome countries and underserved groups in high income-countries, which are experiencing an epidemiologic transition [34].

Because antibiotics have been so successful in controlling acute bacterial infections, long term sustainable solutions that involve the exposome that many populations share such as clean water, sanitation, clean air, proper housing, safe infrastructure, green spaces, employment, and social capital are ignored. Thus, although many people may survive an acute illness, the lack of a safe exposome translates to a continuous exposure to stressors leading to epigenetic changes and nurturing an exposome of causes and risk factors for chronic disease. This could explain why rich and poor are suffering from chronic diseases. Neither rich nor poor, class or ethnicity is spared.

Tertiary health care treats individuals with chronic conditions, but does not address the primary causes, i.e., the harmful exposome to which individuals are exposed. Individuals cannot in themselves create a healthy exposome as this can only be achieved through a societal desire to live in a health-promoting environment. Humans have always lived as groups and built communities for survival. Primary public health must address the safety of the exposome to minimize allostatic load. Social capital has the potential to connect with public policy in response to demands from the community to bring about positive change for rich and poor alike.

Epigenetics provides a means to elucidate points of intersection between the exposome and human health. Data generated from epigenetic studies link the real causes of human disease, which can be found in the exposome. Those studies can be a powerful tool in formulating policies that 
create a health-promoting exposome. To accomplish this, public health must be more inclusive of fields other than biomedical sciences. It will take a concerted effort to bring other disciplines, including commerce, transportation, agriculture, and finance as well as businesses and communities working together to create the safe exposome. Health will be an outcome if included in every policy.

Epigenetic studies have shown that many factors of transient gene expression patterns may underlie the simplistic low-hanging fruit hypotheses [35]. Policy makers must take a transdisciplinary approach that takes into account effective prevention strategies by expanding their scope beyond disease treatment. Only then will we have effective prevention strategies that enhance human health. We cannot change the genetic make-up of humans, but we can work toward preventing environmental triggers, which lead to a devastating cascade of events to express the disease phenotype.

Acknowledgement: Research reported in this publication was supported by the National Institute on Minority Health and Health Disparities of the National Institutes of Health under Award Number P20MD006899. The content is solely the responsibility of the authors and does not necessarily represent the official views of the National Institutes of Health.

Conflict of Interest: The Authors report there are no conflicts of Interest.

\section{Reference}

1. Johnson, N.B.; Hayes, L.D.; Brown, K.; Hoo, E.C.; Ethier, K.A. Cdc national health report: Leading causes of morbidity and mortality and associated behavioral risk and protective factors-united states, 2005-2013. MMWR Surveill. Summ. 2014, 63, 3-27.

2. Meloni, M. The social brain meets the reactive genome: Neuroscience, epigenetics and the new social biology. Front. Hum. Neurosci. 2014, 8, 309-321.

3. Toscano, W.A., Jr.; Sehgal, H.; Yang, E.; Spaude, L.; Bettmann, A.F. Environment-gene interaction in common complex diseases: New approaches. AIMS Mol. Sci 2014, 1, 126-140.

4. Collins, F. The case for a us prospective cohort study of genes and environment. Nature (London) 2004, 429, 475-477.

5. Maher, B. The case of the missing heritability. Nature 2008, 456, 18-21.

6. Ekström, T.J. Epigenetic control of gene expression. BBA-General Subjects 2009, 1790, 845-846.

7. Combs-Orme, T. Epigenetics and the social work imperative. Soc. Work 2013, 58, 23-30.

8. Nakamura, J.; Mutlu, E.; Sharma, V.; Collins, L.; Bodner, W.; Yu, R.; Lai, Y.; Moeller, B.; Lu, K.; Swenberg, J. The endogenous exposome. DNA Repair (Amst) 2014, 19, 3-13.

9. Wild, C.P. The exposome: From concept to utility. Int. J. Epidemiol. 2012, 41, 24- 32.

10. Haugen, A.C.; Schug, T.T.; Collman, G.; Heindel, J.J. Evolution of dohad: The impact of environmental health sciences. J. Dev. Origins of Health and Dis. 2014, 10 (In Press).

11. Guthman, J.; Mansfield, B. The implications of environmental epigenetics: A new direction for geographic inquiry on health, space, and nature-society relations. Prog. Hum. Geogr. 2013, 37, 486-504.

12. Case, A.; Lubotsky, D.; Paxson, C. Economic status and health in childhood: The origins of the health gradient. Am. Econ. Rev. 2002, 92, 1308-1334.

13. Currie, A.; Shields, M.A.; Price, S.W. The child health/ family income gradient: Evidence from england. J. Health Econ. 2007, 26, 213-232.

14. Putnam, R.D. Bowling alone: The collapse and revival of american community. Simon and Schuster: New York, NY, 2000.

15. McPherson, K.M.; Kerr, S.; McGee, E.; Cheater, F.; Morgan, A. The role and impact of social capital on the health and wellbeing of children and adolescents: A systematic review; Glasgow Centre for Population Health: Glasgow, Scotland, 2013; p 86 pp.

16. Whitley, R. Social capital and public health. In Social capital and health, Kawachi, I.; Subramanian, S.V.; Kim, D., Eds. Springer- Verlg: New York, NY, 2007; pp 232-270.

17. Stout, C.; Morrow, J.; Brandt, E.N.; Wolf, S. Study of an italian-american community in pa: Unusually low incidence of death from myocardial infarction. J. Am. Med. Assoc. 1964, 188, 845-849.

18. Egolf, B.; Lasker, J.; Wolf, S.; Potvin, L. The roseto effect: A 50 year comparison of mortality rates. Am. J. Public Health 1992, 82, 1089-1092. 
19. Danaraj, T.J.; Acker, M.S.; Danaraj, W.; Ong, W.H.; Yam, T.B. Ethnic group differences in coronary heart disease in singapore. An analysis of necropsy records. Am. Heart J. 1959, 58, 516-526.

20. Shi, Y.; Zhong, S. From genomes to societies: A holistic view of determinants of human health. Curr. Opin. Biotechnol. 2014, 28, 134-142.

21. Tung, J.; Gilad, Y. Social environmental effects on gene regulation. Cell. Mol. Life Sci. 2013, 70, 4323-4339.

22. Marmot, M.G.; Smith, D.G.; Stansfeld, S.; Patel, C.; North, F.; Head, J.; White, I.; Brunner, E.; Feeney, A. Health inequalities among british civil servants: The whitehall ii study. The Lancet 1991, 337, 1387-1393.

23. Cole, S.W.; Conti, G.; Arevalo, J.M.G.; Ruggiero, A.M.; Heckman, J.J.; Suomi, S.J. Transcriptional modulation of the developing immune system by early life social adversity. Proc. Natl. Acad. Sci. USA 2012, 109, 20578-20583.

24. Miller, G.E.; Chen, E.; Sze, J.; Marin, T.; Arevalo, J.M.G.; Doll, R.; Ma, R.; Sole, S.W. A functional genomic fingerprint of chronic stress in humans: Blunted glucocorticoid and increased nf-k b signaling. Biol. Psych. 2008, 64, 266-272.

25. Conti, G.; Hansman, C.; Heckman, J.J.; Novak, M.F.X.; Ruggiero, A.M.; Suomi, S.J. Proc. Natl. Acad. Sci. USA 2012, 109, 8866-8871.

26. Ball, S.J.; Jacoby, P.; Zubrick, S.R. Socioeconomic statue accounts for rapidly increasing geographic variation in the incidence of poor fetal growth. Int. J. Environ. Res. Public Health 2013, 10, 2606-2520.

27. Meloni, M. The social brain meets the reactive genome: Neuroscience, epigenetics and the new social biology. Front. in Hum. Neurosci. 2014, 8, 12.

28. Nebert, D.W.; Zhang, G.; Vesell, E.S. Genetic risk prediction: Indivualized variability in susceptibility to toxicants. Ann. Rev. Pharmacol. Toxicol. 2013, 53, 355-375.

29. McEwen, B.S. Protective and damaging effects of stress mediators. N. Engl. J. Med 1998, 338, 171-179.

30. Schmidt, C.W. Beyond malnutrition the role of sanitation in stunted growth. Environ. Health Perspect. 2014, 122, A298-A303.

31. Tammen, S.A.; Friso, S.; Choi, S.W. Epigenetics: The link between nature and nurture. Mol. Aspects Med. 2013, 34, 753-764.

32. Feinberg, P. Epigenomics reveals a functional genome anatomy and a new approach to common disesae. Nat. Biotechnol. 2010, 28, 1049-1052.

33. Jirtle, R.L.; Skinner, M.K. Environmental epigenomics and disease susceptibility. Nat. Rev. Genet. 2007, 8, 253-262.

34. McKeown, R.E. The epidemiologic transition: Changing patterns of mortality and population dynamics. Am. J. Lifestyle Med. 2009, 3 (1 Suppl.), 19s-26s.

35. Hirasawa, R.; Fell, R. Genomic imprinting and human disease. Essays in Biochem. 2010, 48, 187-200.

(C) 2017 by the authors. Licensee Preprints, Basel, Switzerland. This article is an open access article distributed under the terms and conditions of the Creative Commons by Attribution (CC-BY) license (http://creativecommons.org/licenses/by/4.0/). 